the incident particles. Recent work (for example, Hughes, Space Res., 14, 789; 1974) indicates that about $95 \%$ of the mass incident on the Earth is in the form of particles of mass $10^{-9}-10^{-5} \mathrm{~g}$ which have very low geocentric velocities and different orbits from those assumed by Mitra.

The second point concerns Mitra's assumption that "the effective deceleration of the velocity initially possessed by a meteoroid takes place in the $150-400 \mathrm{~km}$ region". This is contrary to visual and radar observations of the more massive meteoroids ( $>10^{-5} \mathrm{~g}$ ) which have maximum ablation at heights of about $90 \mathrm{~km}$. Either it must be assumed that the super-rotation is produced by the minor amount of ablation and momentum transfer that occurs at heights above $150 \mathrm{~km}$, in which case Mitra's 34 tons a day influx must be increased accordingly, or a process of momentum transfer from the lower layers where the ablation occurs to the regions of super-rotation must be invoked; similar to that propounded for the transfer of atmospheric wave energy.

It seems therefore that the cause of super-rotation still remains a mystery and that the Earth's spinning upper atmosphere is still the happy hunting ground for new theories.

David W. Hughes

\section{The collagen triple helix}

James Watson, in his Nobel address on the work which led to the Watson-Crick model for DNA, spoke of his fear that this structure might have turned out to be "dull and uninteresting, like collagen". In the event, collagen has turned out to be a most interesting protein which has occupied the attention of workers from a wide range of disciplines.

Recently, attention has been focussed on the intracellular process by which the three polypeptide subunits ( $\alpha$ chains) of monomeric collagen form its characteristic triple-stranded helix. The very slow and incomplete in vitro renaturation of $\alpha$ chains derived from extra-cellular collagen led to the proposal by Speakman (Nature, 229, 241; 1971) that the $\alpha$ chains as initially synthesised might have peptide extensions. It was postulated that these extensions or "registration peptides' might serve to bring the three $\alpha$ chains into a conformation which would allow rapid formation of the triple helix. Subsequently much evidence has accumulated to indicate the existence of a precursor of this type (called procollagen) during the biosynthesis of collagen in a wide variety of tissues, and some of the properties of procollagen have been defined (reviewed by Schofield and Prockop, Clin. Orthopaed. Rel. Res., 97, 175; 1973). Initially considerable controversy existed about the size of these extensions and their amino acid composition, but there now seems to be general agreement that type I and type II procollagens comprise polypeptides of $120,000-125,000$ daltons. Moreover, it seems that all pro- $\alpha$ chains contain cysteine, an amino acid not usually found in extracellular interstitial collagen, with the possible exception of a newly discovered form of collagen (type III) recently isolated from several human tissues (Chung and Miller, Science, 183, 1200; 1974).

The demonstration of the presence of cysteine in procollagen suggested that disulphide bonding occurs between the pro- $\alpha$ chains and several groups have now reported that procollagen is secreted as a triple-stranded disulphidestabilised molecule of molecular weight of about 360,000 daltons. Similar observations have been reported by Monson and Bornstein (Proc. natn. Acad. Sci. U.S.A., 70,
3521 ; 1973) who also demonstrated that the procedures used previously in their laboratory for the extraction of procollagen from cranial bones resulted in partial cleavage of the extension peptides by endogenous proteases such that the regions containing the interchain disulphide linkages were lost. This finding reconciles several apparently conflicting reports concerning the occurrence of interchain disulphide bonds in procollagen.

The possibility of a relationship between the process of disulphide bond formation among pro- $x$ chains and that of triple-helix formation was suggested by studies reported by Grant et al. (J. biol: Chem., 248, 7432; 1973) on the biosynthesis of a basement membrane collagen of embryonic chick lens. Procollagen secreted by lens cells was found to be linked by interchain disulphide bonds and in a triple helical conformation. By contrast, intracellular lens collagen was found to be in a random coil conformation, and interchain disulphide bonds between the pro- $x$ chains were also absent. That the formation of the disulphide bonds may be of considerable importance in promoting the formation of the triple helix is also indicated by studies using pulse label and chase experiments in embryonic chick tendon cells (Schofield et al., Biochemistry, 13, 1801; 1974) and cartilage cells (Uitto et al., Fed. Proc., 33, 617; 1974). A close correlation was observed between the extent of interchain disulphide bonding among pro- $x$ chains and the extent to which the procollagen was in a triple-helical conformation. The rates of these processes, however, seem to vary according to the cell type and may thus influence the time taken for secretion of procollagen from different types of cells.

Further evidence of a role for disulphide bonds in procollagen synthesis comes from the work of Uitto and Prockop (Biochem, biophys. Res. Commun., 55, 904; 1973). Embryonic chick tendon cells were incubated at $37^{\circ} \mathrm{C}$ under conditions in which they synthesised and accumulated protocollagen, (unhydroxylated procollagen) which is nonhelical above $24^{\circ} \mathrm{C}$. When the cells were cooled to $15^{\circ} \mathrm{C}$, the intracellular protocollagen, which was shown to contain interchain disulphide bonds, became triple helical within five minutes. But when disulphide bonds in the amino-terminal extensions of protocollagen were reduced by treating the cells with dithiothreitol, the rate of helix formation on cooling was markedly decreased; this suggests that the formation of interchain disulphide bonds is probably a necessary prelude to helix formation. Whether or not this process is mediated by enzymes is unknown, but in tendon cells interchain disulphide bonding of procollagen polypeptides occurs in the rough endoplasmic reticulum soon after release of the completed polypeptides from membrane-bound ribosomes (Harwood et al., Biochem. biophys. Res. Commun., 55, 1188, 1973).

A further factor in considering the formation of the collagen triple helix is the part played by hydroxyproline. Uitto and Prockop using protocollagen were able to dissociate the disulphide bonding process from any influences exerted by hydroxyproline residues in the polypeptides. It is clear from studies of the denaturation temperatures of procollagen and protocollagen, however, that the absence of hydroxylated prolyl residues markedly decreases the thermal stability of the collagen triple helix (Jimenez et al., Biochem. biophys. Res. Commun., 52, 106; 1973; Berg and Prockop, ibid., 115). It seems therefore that the major role of hydroxyproline is to maintain the procollagen polypeptides in a triple-helical conformation under physiological conditions, and that one of the chief roles of the amino-terminal extensions is to facilitate chain association through the formation of interchain disulphide linkages leading to the formation of the triple-helical structure in a time which is biologically feasible.

D. S. JACKSON

M. E. Grant 